\title{
RESULTADOS DE LA CIRUGÍA ACTUAL PARA EL TRATAMIENTO DEL CÁNCER DE ESÓFAGO*
}

\author{
Drs. Italo Braghetto M. ${ }^{1}$, Gonzalo Cardemil H. ${ }^{1}$, Attila Csendes J. ${ }^{1}$, \\ Enrique Lanzarini S. ${ }^{1}$, Maher Mushle K. ${ }^{1}$, Francisco Venturelli M. ${ }^{1}$, \\ Carlos Mandiola B. ${ }^{1}$, Gonzalo Masia L. ${ }^{1}$, Francesca Gattini S. ${ }^{1}$ \\ 1 Departamento de Cirugía Hospital Clínico de la Universidad de Chile. \\ Santiago, Chile.
}

\begin{abstract}
Surgical treatment of esophageal cancer. an update

Esophageal cancer surgery is a challenge despite the advances in surgical techniques and perioperative care. It continues to have high rates of complications and mortality, even in large volume centers. This paper revises the epidemiology, staging and therapeutic options for esophageal cancer, according to its stage and location. It also encompasses the digestive tract reconstruction options, according to our own experience and reports from abroad.
\end{abstract}

Key words: Esophageal cancer, surgery, digestive tract reconstruction.

\section{Resumen}

La cirugía del cáncer de esófago ha sido y sigue siendo un desafío pese a los avances en técnica quirúrgica y cuidados peri operatorios y a pesar de que las complicaciones postoperatorias y la morbimortalidad sigue siendo alta, aún en centros con alto volumen de procedimientos y más aún en centros no dedicados a la atención de estos pacientes. En este artículo se revisarán algunos aspectos epidemiológicos, las herramientas diagnósticas actuales, su etapificación, y las opciones terapéuticas de acuerdo al estadío, sea en etapas precoces o avanzadas, la localización y tipo de tumor, opciones de reconstrucción del tránsito digestivo en base a lo reportado en la literatura internacional, la experiencia nacional y en base a nuestros propios resultados en el Departamento de Cirugía del Hospital Clínico de la Universidad de Chile.

Palabras clave: Cáncer de esófago, cirugía, tratamiento.

\footnotetext{
*Recibido el 13 de abril de 2015 y aceptado para publicación el 24 de septiembre de 2015.

Los autores no refieren conflictos de interés.

Correspondencia: Dr. Italo Braghetto M. cirugia@braghetto.cl
} 


\section{Introducción}

El cáncer de esófago predominantemente ocurre en las últimas décadas de la vida y la edad media es sobre los 60 años. Si bien se han logrado importantes mejoras en su tratamiento multimodal, la cirugía sigue siendo el pilar del tratamiento curativo de la enfermedad. La esofaguectomía sigue teniendo altas tasas de complicaciones y mortalidad postoperatoria con rangos entre 2 y $6 \%$, incluso en centros especializados. Las técnicas que actualmente se utilizan más frecuentemente son la esofaguectomía transtorácica y la transhiatal ${ }^{1-5}$. Si bien la esofaguectomía abierta transhiatal con ascenso gástrico y anastomosis cervical teóricamente presenta un menor trauma quirúrgico, ofrece limitaciones no menores en cuanto a la factibilidad de resección del tercio medio del esófago junto a una inadecuada disección de linfonodos a este nivel, y aumento del riesgo de lesión de estructuras adyacentes, que pueden alcanzar hasta un $50 \%$ de los casos y una mortalidad entre un 8 y $23 \% \%^{6-8}$.

\section{Epidemiología}

La epidemiología de la enfermedad en el mundo muestra diferencias que es necesario señalar. En nuestro país, en los pacientes con cáncer de esófago en comparación con los pacientes europeos y norteamericanos donde predomina el adenocarcinoma, predomina el cáncer escamoso, el diagnóstico en general es más tardío en etapas más avanzadas, son pacientes de mayor riego operatorio, añosos o desnutridos, en los cuales es posible resección curativa en sólo cerca del $25 \%$ en comparación con la tasa de resección R0 que llega al $60 \%$ en promedio $\mathrm{y}$ que pueden incidir en los resultados finales del tratamiento del cáncer de esófago.

En este artículo revisaremos los aspectos que hasta el momento han sido más o menos consensuados en base a la experiencia de nuestro grupo de trabajo como de la revisión de la literatura internacional revisando los datos publicados en Pubmed, Medline o Cochrane Library.

\section{Diagnóstico}

Respecto de los consensos actuales en cuanto a diagnóstico, hay relativo acuerdo y aceptación de su estudio con los métodos de diagnóstico y etapificación disponibles, pero existen algunas diferencias entre la realidad de nuestro país comparado con los diferentes escenarios observados ya sea en occidente como en los países asiáticos o en Latinoamérica. No hay duda que el arma diagnóstica fundamental es la endoscopia y biopsia y existen varios métodos que son importantes en la etapificación del cáncer de esófago.

\section{Etapificación del cáncer de esófago}

El TC de tórax abdomen y pelvis detecta las metástasis en un $60-90 \%$ de los casos. En cuanto al compromiso en profundidad de la pared esofágica posee una exactitud diagnóstica en la detección de los T4 de un $90 \%{ }^{9}$, sin embargo, tiene bajo rendimiento en diferenciar entre un $\mathrm{T} 1, \mathrm{~T} 2$ y $\mathrm{T} 3{ }^{10}$.

La exactitud diagnóstica del TC para compromiso linfonodal es de un 50-60\%, inferior al PET y a la endosonografía (ES) ${ }^{9}$.

La ES es el método más exacto para la detección locoregional. Tiene una exactitud diagnóstica de un $90 \%$ para el $\mathrm{T}$ y $70-80 \%$ para el $\mathrm{N}^{9}$, que es mejor que la del PET, con una mayor sensibilidad de 51\% en la detección del tumor locoregional ${ }^{11}$. Sin embargo, el PET supera al TC y la ES en la detección de linfonodos más allá de los $5 \mathrm{~cm}$ del tumor primario.

La utilidad del PET es principalmente la detección de metástasis no sospechadas, lo cual sucede en el $5-28 \%$ de los casos ${ }^{11}$.

En el consenso de la Sociedad Latinoamericana de Gastroenterología Oncológica SLAGO 2011 ${ }^{12}$, se estableció que para nuestro país el examen que tiene el mejor rendimiento como examen único, es la TC en un $69 \%$. El esquema propuesto es iniciar el estudio con TC para descartar T4 y M1, luego edosonografía para evaluar con mayor exactitud el $\mathrm{T}$ y el $\mathrm{N}$ y luego el PET para evidenciar la presencia de metástasis no detectadas previamente en los T23, N0-1.

\section{Tratamiento actual}

De acuerdo al estadio del cáncer existen diferentes opciones: ¿Cuándo cirugía endoscópica? ¿En qué pacientes? ¿Es útil la neoadyuvancia conociendo los resultados de la experiencia europea con una tasa de respuesta cercana al 50\%? Siendo la cirugía la única alternativa de curación ¿Cuándo cirugía clásica abierta o mininvasiva? Son puntos que actualmente están en plena discusión en la literatura mundial ${ }^{9,12-14}$.

\section{Tratamiento del cáncer de esófago in situ}

Se han sugerido diferentes opciones de terapia endoscópica como el uso de terapia fotodinámica o ablación con radiofrecuencia que se está utilizando especialmente en paciente con displasia de alto grado de localización mucosa, en la que en Oriente se utiliza más frecuentemente la resección submucosa 
endoscópica. El problema de estas técnicas es que pueden presentar recurrencia local entre 15 a $30 \%$, recurrencia metacrónica en más de un $20 \%$, pueden retrasar una cirugía, por lo que el seguimiento en este caso debe ser muy acucioso tanto con endoscopia, biopsias y endosonografía para detección de recurrencia precoz. La sobrevida a 5 años es de $84 \%$ libre de tumor. En el cáncer escamoso que compromete la muscular de la mucosa la incidencia de compromiso linfonodal es de un $6-18 \%$, por lo que la mucosectomía estaría indicada sólo en tumores que comprometen hasta la mucosa superficial $(\mathrm{m} 1)$, escamosos $<3 \mathrm{~cm}$ que comprometen menos de $3 / 4$ de la circunferencia esofágica ${ }^{14,15}$.

En el adenocarcinoma, el 10\% de los tumores que comprometen la muscular de la mucosa presentan compromiso linfonodal, por lo que también estaría indicada la mucosectomía en la displasia de alto grado o tumores que comprometen sólo la mucosa superficial ${ }^{15}$. Sin embargo, es posible que hasta un $30 \%$ de las displasia de alto grado pueden presentar un cáncer invasor más allá de la muscular de la mucosa y que más del $10 \%$ presente recurrencia de la enfermedad, por lo que estos pacientes deben quedar en estricto seguimiento endoscópico ${ }^{16}$. Debido a la gran morbimortalidad de la esofaguectomía (> 5\%), esta última no estaría indicada en esta etapa de la enfermedad ${ }^{16,17}$.

\section{Tratamiento del cáncer de esófago estadio I}

Está reportado que en el carcinoma mucoso superficial, independiente del tipo histológico, la incidencia de compromiso linfonodal es un $0-5 \%{ }^{15,18,19}$, por lo que se ha planteado la mucosectomía endoscópica en este estadío tumoral. Por el contrario, en los que comprometen la muscular de la mucosa, la incidencia de compromiso linfonodal es mayor al $5 \%$, por lo que estaría indicada la esofaguectomía ${ }^{15}$. Según las guías del NCCN, los tumores que comprometen hasta la muscular de la mucosa pueden ser tratados mediante esofaguectomía o mucosectomía, esta última siempre cuando no tengan compromiso linfonodal, no tengan invasión vascular ni linfática, ni sean mal diferenciados. Otra indicación es cuando se presenta en pacientes que no están en condiciones de ser operados por sus patologías asociadas ${ }^{21}$.

Si bien hay estudios que demuestran buena sobrevida a 5 años mediante mucosectomía $(80 \%$ aproximadamente $)^{20}$, con cirugía la sobrevida puede alcanzar un $100 \%$ a 5 años ${ }^{18,19,21-23}$. Por otra parte, es muy difícil saber cuándo efectivamente un cáncer compromete sólo la mucosa superficial, ya que la endosonografía no presenta una exactitud diagnóstica de un $100 \%$.

En los cánceres con compromiso de la muscular de la mucosa y submucosos la incidencia de compromiso linfonodal es mayor a un $30 \%$ por lo que en estos casos la mucosectomía no estaría indicada ${ }^{19}$.

En el consenso de la Sociedad Latinoamericana de Gastroenterología Oncológica del 2011, al consultarle a los panelistas sobre si para el estadio I el tratamiento de elección era la resección endoscópica o la esofaguectomía, el $54 \%$ votó por la resección quirúrgica. En la mesa de discusión se consensuó que la decisión debe ser dada caso a caso dependiendo de los resultados de los estudios y características generales del paciente. Los tipos de esofaguectomía actualmente en uso son la esofaguectomía transhiatal, la misma técnica pero con preservación del vago ("vagal Sparing") y la esofaguectomía en block, que son técnicas poco difundidas y poco usadas. Actualmente las técnicas mini invasivas vía toracoscópica y laparoscópicas han ganado mucha difusión y muchos grandes centros de excelencia han adoptado esta técnica como la de elección ${ }^{24-26}$.

\section{Tratamiento en cáncer localmente avanzado}

En estos casos se ha observado que presentan linfonodos $(+)$ en un $25 \%$ por lo tanto, surge la discusión respecto del tipo de linfadenectomía, qué tipo de resección efectuar, ya sea en bloque o linfaadenectomía de 3 campos y/o Terapia multimodal + Cirugía ${ }^{27-30}$.

En nuestra experiencia, la adopción de terapia multimodal no ha sido posible de implementar por múltiples razones, fundamentalmente razones de cobertura financiera, oportunidad de atención, características clínicas.

\section{Cáncer de esófago cervical}

El tratamiento quirúrgico de esta localización tumoral es la faringolaringoesofaguectomía, la cual conlleva una altísima morbimortalidad ya que es muy mutilante. El uso de la radioterapia presenta peor sobrevida que la radio-quimioterapia, usando la quimioterapia como radiosensibilizador ${ }^{31} \mathrm{y}$ al evaluar la radio-quimioterapia vs la cirugía radical la sobrevida no varía ${ }^{32,33}$. Existe consenso que para esos tumores la radioterapia+quimioterapia es el tratamiento de elección y las grandes cirugías resectivas son excepcionales.

\section{Cáncer intratorácico: vía de abordaje}

\section{Cáncer de tercio medio}

En el cáncer de tercio medio o superior, supracarinal de acuerdo a los postulados de Siewert, nadie discute que hay que practicar un abordaje torácico. Actualmente la discusión está centrada en la vía por 
toracoscopia o toracotomía. La vía mínimamente invasiva presenta menor respuesta inflamatoria, menos dolor postoperatorio y menor estadía hospitalaria. Seogurakis ${ }^{34}$, publicó un metaanálisis donde incluyó 3 estudios comparativos concluyendo que no había diferencia ni en la morbilidad ni en la sobrevida a 5 años entre ambas técnicas ${ }^{34}$. Sin embargo, en el mismo metaanálisis se incluyeron 5 estudios donde se compara la cirugía completamente mini invasiva (torácica y abdominal) vs la vía abierta (toracotomía y laparotomía), evidenciándose que la vía mínimamente invasiva presentó en general menos complicaciones con similar mortalidad operatoria y sobrevida a 3 años. En nuestra opinión ${ }^{35}$, la cirugía mínimamente invasiva es factible, los resultados técnicos y de pronóstico son comparables y en cuanto a sobrevida no hay evidencias categóricas de superioridad entre ambos abordajes. En caso de que el paciente hubiese sido sometido a neoadyuvancia, la técnica toracoscópica no es recomendable pues puede aumentar las dificultades en la disección del tumor de las estructuras vecinas. Aunque este punto está en estudio.

En Chile, el 50\% de los cirujanos dedicados al tratamiento del cáncer de esófago, escogen la alternativa toracoscópica, para la esofaguectomía ${ }^{12}$.

\section{Cáncer de esófago de tercio inferior}

En el cáncer esofágico de tercio inferior la discusión se centra en cuanto a su vía de abordaje. En relación a esto existe un meta análisis que compara la vía transhiatal con la transtorácica.

Hulscher en su publicación incluyó 50 estudios, 24 estudios comparativos, 3 estudios prospectivos comparativos, 3 estudios prospectivos aleatorizados y 18 retrospectivos, el resto de los estudios fueron serie de casos, o de la via transhiatal o de la vía transtorácica ${ }^{36}$. La vía transtorácica presentó una mayor incidencia de complicaciones pulmonares (RR: 1,47. CI: 1,29-1,68), quilotórax (RR: 1,7. CI: 10,52,7 ), estadía hospitalaria (21 vs 17,8 días; $\mathrm{p}<0,05$ ) y mortalidad 9,2 vs 5,7\% (OR 1,60, 95\% CI 1,89, 1,35). La vía transhiatal presentó más parálisis de las cuerdas vocales (OR: 0,36. CI: $0,27-0,47$ ) y filtraciones de la anastomosis (OR: 0,53. CI: 0,45-0,63). Ambas vías presentan similar sobrevida a 5 años. Al analizar sólo los 3 ensayos clínicos en conjunto, no se detectó ninguna diferencia en cuanto a la morbimortalidad. Al analizar el estudio de Orringer et $\mathrm{al}^{30}$, en 2.000 esofaguectomías transhiatales en el último período llegó a tener $1 \%$ de mortalidad, $2 \%$ de lesión del nervio recurrente y $9 \%$ de fístula cervical. Al consultar a los cirujanos de nuestro país sobre qué vía es la de elección para el abordaje del cáncer esofágico del tercio inferior, el 91,7\% optó por la vía transhiatal. Una herramienta para dismi- nuir riesgos de complicaciones es el apoyo de la vídeo asistencia ${ }^{12}$.

\section{Tratamiento según estadio evolutivo}

\section{Cáncer de esófago estadío II y III: tipo de cirugía y lugar para la neoadyuvancia}

Por medio de los resultados de varios estudios se conoce que ni la cirugía ni la RDQMT por si solas logran una sobrevida $>30 \%$ a 5 años ${ }^{1,2,19}$. Sin embargo, hay varios estudios, algunos de ellos prospectivos y randomizados que demuestran una mejor sobrevida al utilizar radio-quimioterapia (RDQMT) preoperatoria, alcanzando un $40 \%$ a 5 años $^{3,20}$. Al tener el tumor T4 compromiso de estructuras vecinas, no tendría indicación de cirugía, al menos que luego de la neoadyuvancia, se convierta en T3 o menos. Otra opción para el T4 es ofrecerle RDQMT sola ya que se podría mejorar la sobrevida a 5 años.

En los T4 con compromiso de vía aérea no se les puede administrar RDT por lo que tendría indicación sólo de endoprótesis.

Al preguntar a los presentes entre qué preferían si cirugía sola o neoadyuvancia más cirugía, el $90 \%$ se inclinó por la segunda opción para el estadio II y el $96 \%$ para el estadio III. Sin embargo, esto en la práctica es difícil de realizar debido a que nuestra población con cáncer de esófago a diferencia de las de Europa y USA, presenta un menor grado de nutrición y de performance status, por lo que el dar esta terapia puede implicar que el paciente no llegue en condiciones adecuadas a la cirugía. Según las guías de la $\mathrm{NCCN}^{21,22}$, a este grupo de pacientes (T2-T4a) idealmente se le debe ofrecer RDQMT preoperatoria, pero también se puede realizar quimioterapia preoperatoria en los adenocarcinomas o sólo esofaguectomía cuando son tumores mayores. En la Figura 1 se muestra un algoritmo sugerido por las guías de manejo en cáncer de esófago del NCCN 2012, menores de $2 \mathrm{~cm}$ bien diferenciados ${ }^{22}$. Este es aún un punto en discusión en centros japoneses.

\section{Cáncer de esófago estadío IV}

En el manejo de este estadío existen múltiples posibilidades planteadas en el consenso: Cirugía paleativa más RDQMT, paliación endoscópica, RDQMT sola, tratamiento individualizado según decisión de comité. En general la cirugía en esta etapa no tiene indicación salvo si ocurre una perforación esofágica.

La RDQMT no tiene beneficio en la sobrevida en pacientes M1.

El tratamiento que se debe efectuar en esta etapa es una decisión difícil porque depende de cada caso. Por ejemplo, es muy distinto el paciente que está en afagia del que no. En el paciente con disfagia o afa- 


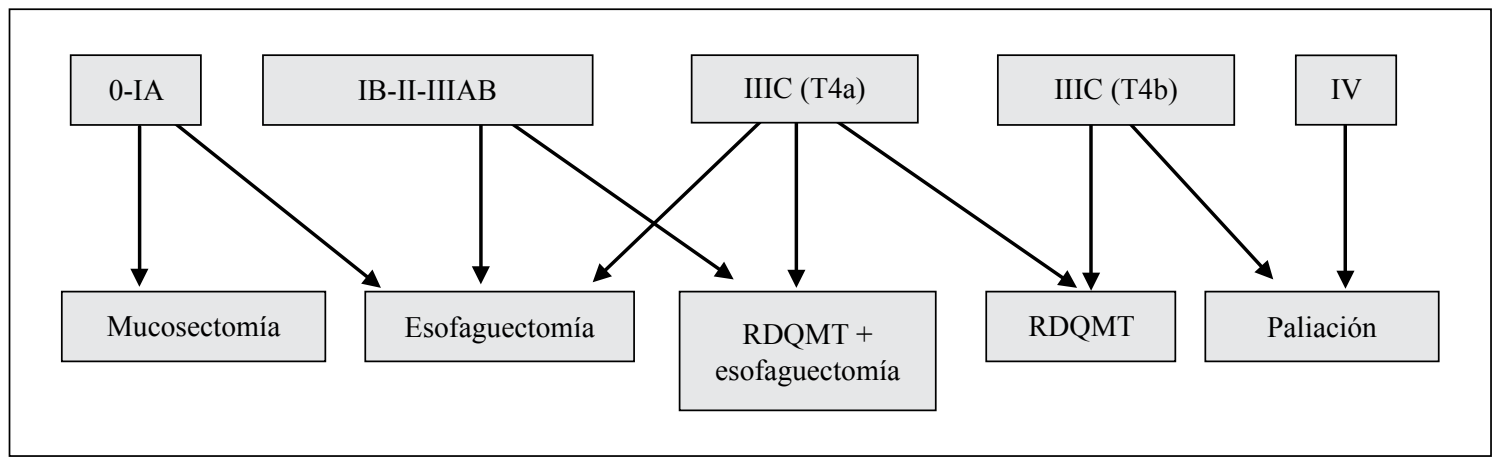

Figura 1.

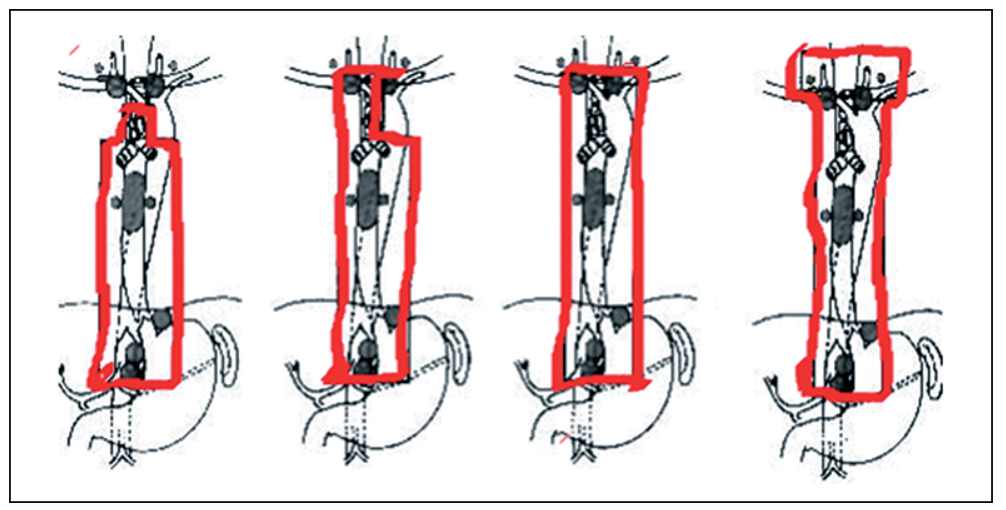

Figura 2. Linfadenectomía de 2 y 3 campos.

gia, la braquiterapia sola demora demasiado tiempo en lograr una regresión del tamaño tumoral, por lo que en estos pacientes está indicado la endoprótesis en una primera instancia ${ }^{37}$, y en caso contrario podría realizarse sólo braquiterapia. Por último si un paciente está en afagia y no está en condiciones de tolerar una RDT sólo se le puede ofrecer manejo del dolor. Si bien el uso de gastrostomía o yeyunostomía puede alimentar al paciente, estas no son del todo aconsejables, ya que una vez que se obstruye completamente el lumen esofágico se hace muy difícil para la familia el manejo de la sialorrea, provocando repetidas hospitalizaciones por obstrucción o infecciones de la vía respiratoria, alargando más la agonía del paciente y de su entorno familiar con una mala calidad de vida. En nuestro país hay bastante experiencia con el uso de endoprótesis como método paliativo de elección. El 83\% de los cirujanos en Chile se inclinan por la idea de que el tratamiento debe ser individualizado según decisión de comité.

\section{Linfadenectomía en cáncer de esófago}

Existen 3 o 4 tipos de linfadenectomía en cáncer de esófago, la linfadenectomía clásica de 2 campos y algunos autores japoneses agregan la de 3 campos con disección ganglionar a nivel cervical (Figura 2) ${ }^{38}$ A su vez la linfadenectomía en 2 campos se subdivide en 3 tipos $^{21}$ : standard, extendida y total.

1. Standard: Linfadenectomía del mediastino inferior y carinales sin resección linfonodal del mediastino superior.

2. Extendida: Linfadenectomía torácica standard + resección linfonodal sólo del lado derecho del mediastino superior.

3. Total: Linfadenectomía standard + resección linfonodal bilateral del mediastino superior.

A diferencia de otras vísceras, la diseminación linfática del esófago no sigue pedículos vasculares y al comprometer la muscular de la mucosa rápidamente se disemina por esta vía. El $10 \%$ de los tumores de tercio inferior presenta compromiso linfonodal en el tercio torácico superior y el $31 \%$ de los de tercio torácico superior presentan compromiso linfonodal perigástrico ${ }^{29}$. En una revisión del año 2003 sobre diferentes trabajos publicados hasta ese momento, se concluyó que la linfadenectomía en 3 campos lograba aumentar la sobrevida y disminuir 
la recurrencia locoregional, pero debía ser realizada en manos experimentadas ${ }^{39}$.

En Chile, un grupo de cirujanos son proclives a efectuar linfadenectomía, juega un rol relevante en el tratamiento del carcinoma espinocelular de esófago pero la evidencia apoya la esofaguectomía subtotal con linfadenectomía en tres campos aún es discutible ${ }^{12,29}$. En adenocarcinoma de tercio inferior, Siewert tipo I, se preconiza una resección de Ivor Lewis con linfadenectomía en dos campos, extendida. Se hace necesario que un estudio prospectivo aleatorizado de respuesta definitiva. Sin embargo, en el mundo no hay consenso al respecto y se hace sólo linfadenectomía de 2 campos.

Al discutir el tema entre los cirujanos que asistieron al consenso, se concluyó que en Chile actualmente no existía ningún centro que cuente con la casuística necesaria como para lograr llevar a cabo una linfadenectomía en 3 campos con baja mortalidad (<5\%). Por lo que hasta el momento sólo el $15 \%$ de los cirujanos se inclinan por la linfadenectomía en 3 campos, por el contrario el 51\% efectúan linfadenectomía de 2 campos extendida y el 34\% por la cirugía con linfadenectomía standard.

\section{Tipo de esofaguectomía en bloc o clásica}

Al igual que en el punto anterior, a pesar de que hay estudios que muestran una mejor sobrevida con la cirugía en bloc (resección de grasa mediastínica posterior con los linfonodos, resección de conducto torácico, pericardio posterior, ambas pleuras, vena ácigos y membrana freno esofágica y parte del anillo hiatal) para los tumores del tercio inferior comparada con cirugía clásica más linfadenectomía en 2 campos total) ${ }^{26,29}$, la primera conlleva más horas quirúrgicas, más días de ventilación mecánica y más días de hospitalización ${ }^{27}$. No existe en este momento ningún centro en Chile con la experiencia suficiente (> 20 esofaguectomías al año $)^{40,41}$, como para llevar a cabo esta técnica con baja mortalidad y el $91 \%$ de los cirujanos realizan la cirugía clásica.

\section{Órgano para reconstrucción del tránsito}

En las esofaguectomías por patología benigna el órgano que se utiliza para la reconstrucción del tránsito es el colon, debido a que éste no se asocia a la presencia de reflujo y úlceras, preserva la función gástrica, la fístula esófago colónica presenta mayor facilidad en su manejo ya que posee una mejor función peristáltica (el estómago ascendido perdería su función). Sin embargo, en cáncer de esófago se prefiere el estómago, debido a que: 1) Al tener esta enfermedad una baja sobrevida a 5 años la función motora deja de ser relevante; 2 ) Sólo requiere de una anastomosis, no 2 o 3, lo que simplifica la cirugía, disminuye el tiempo operatorio y el riesgo de com- plicación postoperatoria; 3) Posee buena irrigación (menor incidencia de necrosis $1-2 \%$ vs $3-10 \%)^{42}$. En Chile, más del 90\% de los cirujanos usan el estómago como órgano de reconstrucción del tránsito.

\section{Vía de reconstrucción del tránsito}

Las vías de reconstrucción más utilizadas son la retroesternal y la transmediastinal. Cada una tiene ventajas y desventajas. La retroesternal tiene la ventaja de que si se necrosa el estómago no se produce una mediastinitis, se puede dar RDT en el postoperatorio si se requiere, en caso de recidiva local no compromete el órgano ascendido, y presenta una mayor facilidad en el manejo de la fístula esófagogástrica. Las desventajas son que presenta una mayor incidencia de fístulas de la anastomosis esófagogástrica cervical, el trayecto de la vía de ascenso es más largo, posee una peor función motora y requiere de la disección de otro plano ${ }^{42}$. La vía mediastínica es más corta, no necesita de la construcción de un túnel adicional y la anastomosis se efectúa en cabos mejor irrigados con menos incidencia de fístula pero tiene más complicaciones cardiorespiratorias y más retención gástrica que el ascenso retrosternal. Una fístula intratorácica tiene más riesgos y presenta mayor mortalidad. El $60 \%$ de los cirujanos se inclinan por la vía retroesternal.

\section{Tratamiento del adenocarcinoma de Barrett}

Hasta ahora la cirugía antirreflujo si bien es capaz de regresar la displasia de bajo grado, no ha logrado demostrar que disminuya la incidencia de cáncer esofágico. Frente a una displasia de alto grado, al menos el $17 \%$ presenta un adenocarcinoma ${ }^{43}$. En el adenocarcinoma mucoso hasta el $6 \%$ presenta compromiso linfonodal ${ }^{31}$. En un paciente que se opera por adenocarcinoma precoz T1n0M0, el no resecar toda la metaplasia intestinal se asocia al desarrollo de una nueva neoplasia hasta en un $30 \%{ }^{44}$. Debido a que es muy difícil definir exactamente hasta donde se encuentra este epitelio y el riesgo de compromiso linfonodal mencionado previamente, se debe resecar todo el esófago. Sin embargo, en caso de que el tumor mida menos de $2 \mathrm{~cm}$ podría intentarse una mucosectomía endoscópica ${ }^{44,45}$. Una opción intermedia entre la esofaguectomía total y la mucosectomía endoscópica es la esofaguectomía distal.

El adenocarcinoma tal como en todo el mundo, también en Chile, muestra un en rápido ascenso en su frecuencia y está fuertemente asociado al esófago de Barrett. En cuanto al tratamiento la resección en etapas precoces es potencialmente curativa. La 
esofaguectomía está indicada en carcinoma submucoso y es la técnica de elección. La esofaguectomía subtotal con fundectomía y disección linfonodal está indicada en cáncer avanzado, ya sea por vía transtorácica, transhiatal o toracolaparoscópica, dejando la selección del abordaje a criterio de la experiencia del grupo quirúrgico.

En Chile, el $61 \%$ se inclina por la esofaguectomía distal y el resto por la esofaguectomía total.

\section{Cirugía mini-invasiva}

El abordaje con procedimientos mini-invasivos es la vía de abordaje que más ha crecido en la última década ${ }^{46}$. En nuestra experiencia con cirugía mini-invasiva, un total de 69 pacientes han sido sometidos a esofaguectomía mínimamente invasiva en un período de 10 años $^{47}$. De los 69 pacientes, 2 $(2,89 \%)$ se encontraban en estadio 0 , mientras que $7(10,14 \%)$ fueron incluidos en estadio I $(\mathrm{Ia}=3$, $\mathrm{Ib}=4), 23(33,33 \%)$ en estadio II (IIa=6, IIb=17), 34 $(49,27 \%)$ en estadio III (IIIa=17, IIIb=7, IIIc=10) y $1(1,44 \%)$ paciente en estadio IV. En relación a la terapia oncológica adyuvante, sólo 2 pacientes $(2,89 \%)$ recibieron tratamiento neoadyuvante (un paciente en estadio IIa se trató con quimio y radioterapia preoperatoria, y otro en etapa IIIa con sólo quimioterapia previo a cirugía). La quimio-radioterapia adyuvante post cirugía fue indicada en etapas II y III según características clínicas y patológicas en 7 pacientes $(10,14 \%)$, de los cuales 5 recibieron quimio-radioterapia adyuvante y 2 sólo quimioterapia postoperatoria. Desde el punto de vista quirúrgico, un total de $39(56,52 \%)$ pacientes fueron sometidos a esofaguectomía transtorácica vídeo asistida, mientras que en $30(43,47 \%)$ pacientes la resección esofágica fue lograda vía transhiatal mínimamente invasiva. La tasa de conversión a cirugía abierta fue de $1,44 \%$ correspondiente a un caso debido a hemorragia secundaria a lesión de vena ácigos y vena pulmonar. En relación a la técnica de reconstitución de tránsito, el procedimiento de elección efectuado en el mismo tiempo quirúrgico tras la esofaguectomía fue el ascenso gástrico tubulizado con anastomosis esófago-gástrica cervical, el que se realizó en 64 pacientes $(92,75 \%)$, mientras que el ascenso de colon se efectuó en 5 casos $(7,24 \%)$. Basadas en el reporte patológico, la mayoría de las resecciones quirúrgicas lograron ser R 0 con un total de 52 pacientes correspondientes al $75,36 \%$ de los casos, mientras que fueron $\mathrm{R} 1$ en 6 pacientes $(8,69 \%)$ y R 2 en 1 paciente $(1,44 \%)$. En $10(14,49 \%)$ pacientes no fue posible precisar la presencia de tumor residual. Del total de pacientes operados el estudio patológico demostró presencia de carcinoma escamoso en 32 (46,37\%) casos, demostrándose la presencia de adenocarcinoma en 37 pacientes $(53,62 \%)$. Un número promedio de 17,17 \pm 9,62 linfonodos (rango de 2-46) fueron resecados por paciente (Tabla 1 ).

En un intento de evaluar la morbilidad en forma estandarizada, se efectuó un análisis de las complicaciones postoperatorias mediante la clasificación de Clavien-Dindo, la que estratifica la gravedad de la complicación en función del tratamiento demandado. De esta manera, se advierte que en $11(15,94 \%)$ pacientes la evolución postoperatoria fue favorable y/o requirió un manejo farmacológico básico, mientras que $33(47,82 \%)$ pacientes presentaron un espectro de complicaciones cuyo manejo fue médico, sin necesidad de procedimientos invasivos que hayan afectado significativamente su evolución postoperatoria (Tabla 2). No obstante, un grupo de pacientes requirió intervenciones quirúrgicas, endoscópicas y/o radiológicas, siendo reoperados un total de $9(13 \%)$ pacientes de nuestra serie. La causa más frecuente de reintervención correspondió a derrame pleural complicado y/o empiema postquirúrgicos

Tabla 1. Técnicas de esofaguectomía mini-invasiva empleadas en el tratamiento y resultados anatomo patológicos

\begin{tabular}{|lc|}
\hline & n pacientes $(\mathbf{\%})$ \\
Técnica de esofaguectomía & \\
Toracoscópica & $39(56,52 \%)$ \\
Transhiatal & $30(43,47 \%)$ \\
Reconstitución de tránsito & $64(92,75 \%)$ \\
Ascenso gástrico tubulizado & $5(7,24 \%)$ \\
Ascenso de colon & $1(1,44 \%)$ \\
Conversión a cirugía abierta & \\
Resección & \\
R0 & $52(75,36 \%)$ \\
R1 & $6(8,69 \%)$ \\
R2 & $1(1,44 \%)$ \\
Rx & $10(14,49 \%)$ \\
Tipo histológico & $37(53,62 \%)$ \\
Adenocarcinoma & $32(46,37 \%)$ \\
Carcinoma escamoso & $17,17 \pm 9,62(2-46)$ \\
Número de linfonodos resecados & \\
Etapa & \\
0 & $11,00 \pm 7,07$ \\
IA & $7,33 \pm 5,03$ \\
IB & $19,50 \pm 4,16$ \\
IIA & $6,66 \pm 5,17$ \\
IIB & $17,29 \pm 10,36$ \\
IIIA & $20,00 \pm 11,27$ \\
IIIB & $18,85 \pm 5,26$ \\
IIIC & $22,30 \pm 7,28$ \\
IV & 13,00 \\
\hline
\end{tabular}


Tabla 2. Complicaciones perioperatorias según clasificación estandarizada de Clavien-Dindo

\begin{tabular}{|lc|}
\hline Grado & $\mathbf{n}(\mathbf{\%})$ \\
I & $11(15,94 \%)$ \\
II & $33(47,82 \%)$ \\
IIIA & $4(5,79 \%)$ \\
IIIB & $6(8,69 \%)$ \\
IVA & $4(5,79 \%)$ \\
IVB & $8(14,49 \%)$ \\
V & $3(4,34 \%)$ \\
\hline
\end{tabular}

Tipo I: Seroma, náuseas y/o vómitos. Tipo II: Fístula de bajo débito, estenosis, neumonía, atelectasia, derrame pleural, colección, parálisis cordal, trombosis venosa profunda, fibrilación auricular, infección urinaria, íleo paralítico, delirium, crisis hipertensiva, insuficiencia renal aguda. Tipo IIIA: Estenosis severa, dilatación gástrica, hidroneumotórax. Tipo IIIB: Fístula de alto débito, empiema pleural, absceso para esofágico, evisceración, lesión vía aérea, lesión de vena ácigos, quilotórax. Tipo IV: Shock séptico foco respiratorio y/o urinario, síndrome disfunción orgánica múltiple, embolia séptica, mediastinitis. Tipo V: Muerte.

( $\mathrm{n}=5$ pacientes), un paciente con lesión de bronquio fuente izquierdo y de conducto torácico con quilotórax secundario, un paciente con hemotórax por lesión de vena innominada, una evisceración contenida y un absceso paraesofágico. La complicación postoperatoria más frecuente corresponde a la fístula de la anastomosis cervical presentándose en $44(63,7 \%)$ pacientes, sin embargo, sólo 2 de ellas correspondieron a fístulas de alto débito que requirieron re-exploración quirúrgica. El resto de los pacientes con fístula $(n=42)$, representan hallazgos radiológicos o fístulas de bajo débito sin mayor significado clínico cuyo manejo fue conservador. En relación a las complicaciones tardías, la estenosis de la anastomosis cervical es la más frecuente con una incidencia de $16(23,18 \%)$ pacientes. La estadía hospitalaria de los pacientes analizados fue $26,78 \pm$ 19 días (Tabla 3).

La mortalidad perioperatoria (complicación tipo $\mathrm{V}$ de Clavien Dindo), se presentó en 2 casos $(2,8 \%)$ (Tabla 3). Entre las causas de muerte en el contexto de la intervención quirúrgica, se encuentra un paciente por mediastinitis aguda secundaria a la perforación del órgano ascendido y un segundo paciente por complicaciones médicas sobreagregadas a un quilotórax y a la lesión del bronquio fuente izquierdo durante su reoperación por quilotórax.

La sobrevida global durante el período de seguimiento de nuestra serie de pacientes sometidos a
Tabla 3. Complicaciones postoperatorias

\begin{tabular}{|lc|}
\hline Complicaciones médicas & \\
Respiratorias & $16(23,2 \%)$ \\
Cardiovasculares & $10(14,5 \%)$ \\
Tromboembolismo & $6(8,6 \%)$ \\
Complicaciones quirúrgicas & \\
Fístula anastomótica & $44(63,7 \%)$ \\
$\quad$ Mayores & $2(2,8 \%)$ \\
$\quad$ Menores & $42(60,8 \%)$ \\
Hemorragia & $2(2,8 \%)$ \\
Mediastinitis & $2(2,8 \%)$ \\
Re operaciones & $9(13 \%)$ \\
Mortalidad & $2(2,8 \%)$ \\
Complicaciones tardías & \\
Estenosis & $16(23,2 \%)$ \\
\hline
\end{tabular}

esofaguectomía por cáncer con técnicas mínimamente invasivas es de $22,59 \pm 25,38$ meses, alcanzando una probabilidad de sobrevida a 3 años de aproximadamente un 30\% (Figura 3). Al analizar la sobrevida de los pacientes por tipo histológico del cáncer, se observa que no existen diferencias significativas entre los pacientes con adenocarcinoma y carcinoma escamoso, siendo muy similar durante los primeros años de seguimiento postoperatorio $\mathrm{p}=0,45 ; \mathrm{Ha}$ zard ratio $=0,7740$; IC 95\% $(0,3952-1,5161)(\mathrm{Fi}-$ gura 4). No obstante, tales diferencias de sobrevida sí se aprecian en relación a las etapas clínicas en que fueron operados los pacientes $(\mathrm{p}=0,0052)$, siendo mayor la probabilidad de sobrevida en etapas más precoces (Figura 5). Estas curvas de sobrevida son muy similares a las obtenidas por nuestro grupo en la era de la cirugía abierta ${ }^{46}$.

Recientes publicaciones confirman la alta tasa de complicaciones y mortalidad postopearatoria oscilando entre 27 y $23 \%$ dependiendo del volumen hospitalario de operaciones anuales ${ }^{47-52}$. Durante las últimas 2 décadas, los enfoques mínimamente invasivos han ganado más adeptos para la realización de varios procedimientos quirúrgicos para el tratamiento de enfermedades tanto benignas como malignas $^{50-52}$. Las técnicas de cirugía mínimamente invasiva descritas para el tratamiento del cáncer esofágico corresponde a la esofaguectomía vídeoasistida más tubulización gástrica abierta o laparoscópica lo que permite la obtención de una mejor visión del campo operatorio en el mediastino, evitando una disección a ciegas y reduciendo el riesgo de complicaciones intraoperatorias y postoperatorias, permitiendo de este modo una mejor visibilidad en la disección de linfonodos paraesofágicos y una mejor etapificación postoperatoria. Por otro lado, la esofaguectomía 

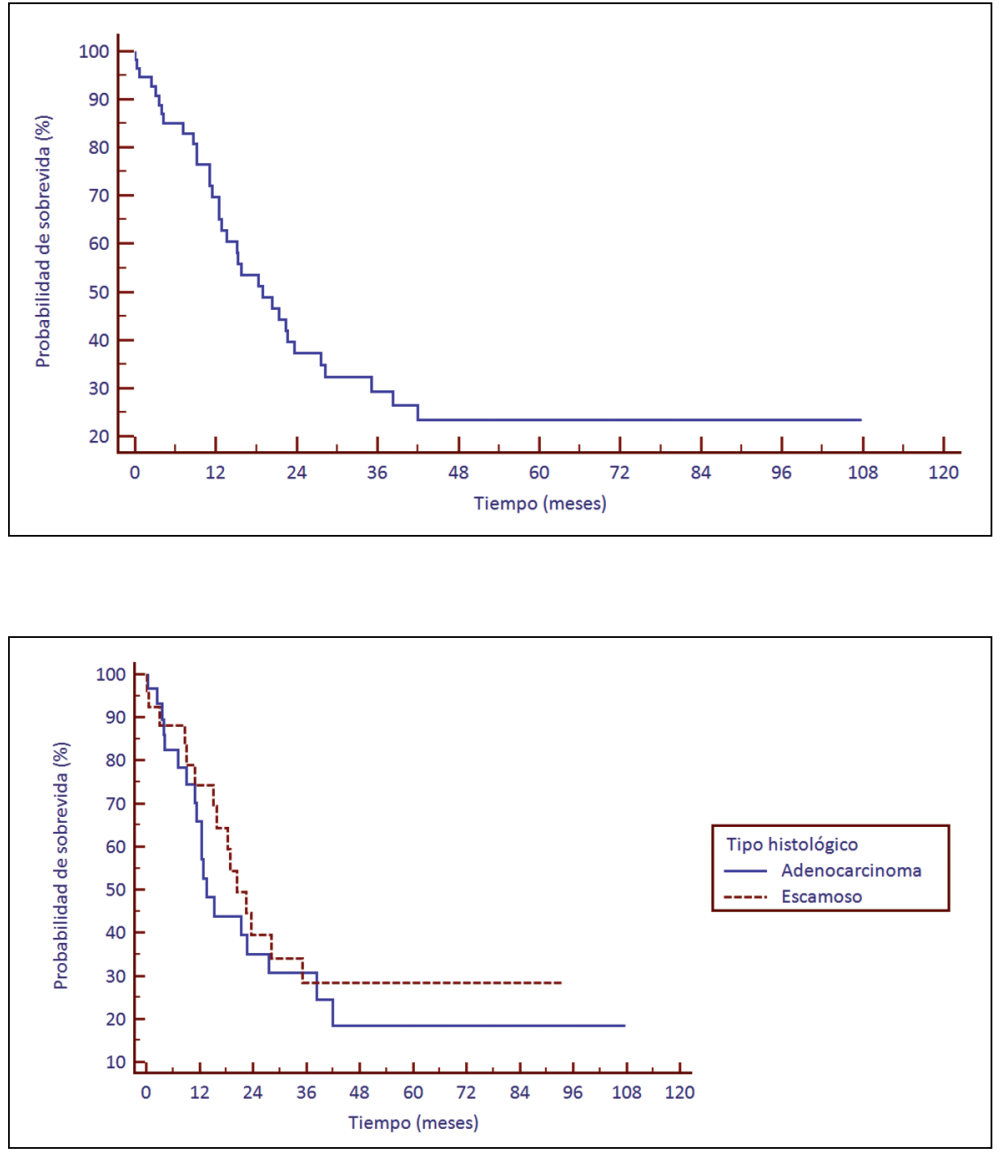

Figura 3. Sobrevida general en esofaguectomía mínimamente invasiva por cáncer de esófago $(n=69)$.
Figura 4. Sobrevida de pacientes sometidos a esofaguectomía mínimamente invasiva según tipo histológico del cáncer. $\mathrm{p}=0,45$; Hazard ratio $=$ 0,7740; IC 95\% (0,3952-1,5161).

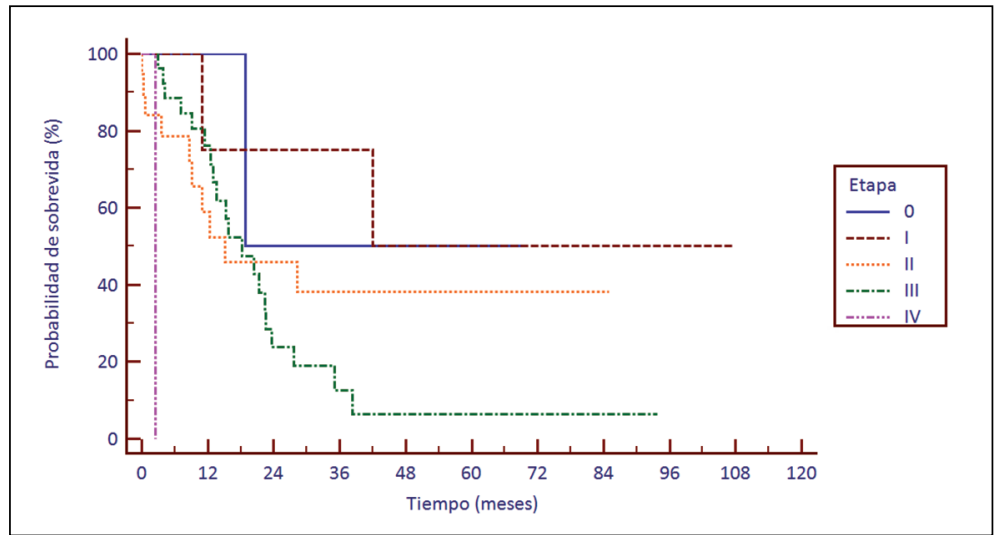

Figura 5. Sobrevida de pacientes sometidos a esofaguectomía mínimamente invasiva según etapa clínica $(\mathrm{n}=69), \mathrm{p}=0,0052$. transtorácica o transhiatal más tubulización gástrica laparoscópica, ha reportado resultados comparables a la técnica con mejor evolución postoperatoria y menor tasa de complicaciones sin constituir un menoscabo en la mortalidad inmediata y en la sobrevida a 5 años plazo $^{53,54}$.
$\mathrm{Al}$ analizar los resultados observados post introducción de la cirugía mini-invasiva en cáncer de esófago inmediatamente surgen algunas preguntas: $¿$ Tiene impacto en las complicaciones y mortalidad postoperatoria? ¿Cuál es la proporción de resecciones R0? ¿La recolección de linfonodos es la misma? 
y finalmente, ¿Tiene repercusión en la sobrevida? Estos son los puntos que analizaremos en esta discusión a la luz de los resultados obtenidos por nuestro grupo y los resultados publicados hasta el momento en la literatura internacional. En los últimos años existen muchos reportes tanto por autores orientales como occidentales evaluando estos puntos ${ }^{53-59}$.

Analizando los resultados, nuestra casuística actual con cirugía mini-invasiva muestra una tasa de mortalidad inferior comparado con nuestros resultados históricos observados durante la era de la cirugía transtorácica o transhiatal abierta ${ }^{23} \mathrm{y}$ también en comparación al metanálisis de Hulscher et al. ${ }^{25,36}$, y también, lo que se encuentra en conformidad con lo mostrado en otros estudios con mayor volumen de pacientes como es el de Luketich et al., con 222 pacientes, alcanzando una mortalidad de un $1,3 \%$, muy por debajo de la mortalidad por esofaguectomía abierta $^{53,54}$. Cabe señalar que la introducción de las técnicas quirúrgicas minimamente invasivas han permitido reducir significativamente la mortalidad perioperatoria de nuestras propias series históricas de pacientes sometidos a abordaje abierto que correspondían a un $11,8 \%$ y $8,6 \%$ para la vía transtorácica y transhiatal abierta respectivamente. Además, desde su implementación en nuestra primera serie de pacientes tratados con cirugía mínimamente invasiva en esofaguectomía durante el período 1993-2003, hemos logrado reducir la tasa de mortalidad desde un $6,4 \%$ a un $2,8 \%$ en los últimos 5 años $^{8}$. En la serie actual, desde el punto de vista de complicaciones y morbilidad postoperatoria, la complicación precoz más importante fue la dehiscencia de la anastomosis cervical. El 2,89\% de los pacientes requirieron de reintervención quirúrgica por fístulas de alto débito, resultado comparable a otros estudios y metaanálisis que van desde el $4-11,7 \%$, con un promedio de un $7,7 \% \%^{53,58-61}$. Por otro lado, la complicación tardía más frecuente fue la estenosis de la anastomosis cervical en el $23,18 \%$ de los pacientes, resultados comparables a los mostrados por Henriques et al..$^{61}$, en pacientes sometidos a esofaguectomía debido a cáncer y a megaesófago avanzado, con un $24 \%$ de incidencia a 36 meses de seguimiento. La tasa de conversión a cirugía abierta en nuestra serie fue de un $1,44 \%$ correspondiente a un paciente con lesiones vasculares de venas ácigos y pulmonar, tasa comparativamente menor a otras series como la de Luketich con un 7,2\% de conversiones ${ }^{53}$. Al revisar la literatura observamos que las complicaciones observadas por nuestro grupo son bastante similares a lo publicado por diversos autores en Europa USA y en países orientales ${ }^{60-64}$.

Warner ${ }^{56}$, en un reciente trabajo reporta un $40 \%$ de complicaciones menores y $30 \%$ de complicaciones mayores destacando reoperaciones en un $7 \%$, hemorragia $1,6 \%$, fístula en un $14 \%$, complicaciones respiratorias $3 \%$, y estenosis hasta en un $20 \%$. Nosotros hemos observado un porcentaje mayor de fístula pues en más del $90 \%$ de los casos efectuamos anastomosis cervicales que presentan mayor riesgo de fístulas en comparación con anastomosis intratorácicas, pero la mortalidad actual está tan baja como la reportada por diferentes grupos, la cual oscila entre un $2-5 \%{ }^{61,65}$. En una revisión sistemática incluyendo más de 1.100 pacientes comparando la vía abierta con la cirugía mini-invasiva, se confirma que esta última se asocia a menor morbimortalidad menor estadía hospitalaria aún en pacientes sometidos previamente a quimio radiación por tumores avanzados, sin que hubiera un incremento en la morbimortalidad operatoria y la sobrevida no parece haberse comprometido por el uso de cirugía mini-invasiva ${ }^{56}$.

Se podría pensar que la técnica de abordaje mini-invasivo podría eventualmente tener resultados deletéreos en cuanto a la sobrevida de los pacientes. Sin embargo, los resultados obtenidos en cuanto a porcentaje de pacientes con resección R0 y la recolección de linfonodos han demostrado ser iguales o mejores que la cirugía por abordaje clásico, lo que obviamente tiene directa incidencia en la sobrevida de los pacientes. Recientemente hay excelentes reportes dando cuenta de los resultados en cuanto a sobrevida y la tendencia es aceptar que la cirugía mini-invasiva no compromete los resultados oncológicos $^{65-69}$.

Desde el advenimiento de la cirugía mínimamente invasiva, la adecuada resección oncológica en comparación a la cirugía abierta ha sido cuestionada en relación a los márgenes de resección, la completa linfadenectomía, la correcta etapificación postoperatoria y la sobrevida a largo plazo ${ }^{37,39}$. Todas las resecciones oncológicas potencialmente curativas, requieren de un margen completo de resección del tumor primario R0, siendo éste el factor de mayor importancia en la sobrevida de los pacientes. Si bien es cierto, que inicialmente la esofaguectomía por técnica mínimamente invasiva se reservó para estadios tempranos, actualmente se ha extendido su indicación para estadios más avanzados en función de los recientes resultados. Nuestro grupo logró un nivel de resección R0 en el 75,36\% de los pacientes $y$ en el $14,49 \%$ no fue posible detectar presencia del tumor residual, probablemente en el contexto del tratamiento neoadyuvante, teniendo márgenes positivos en el $10,7 \%$ de los pacientes, resultados comparables a los obtenidos por técnica quirúrgica abierta como los reportados por Smithers et al., con un 18,9\% de márgenes positivos ${ }^{58}$. Del mismo modo, Thomson et al., logró demostrar que no existía diferencia significativa en los niveles de recurrencia del tumor en función del nivel de resección por ambas vías de 
abordaje, abierto o mínimamente invasivo, resultados comparables a los de nuestra serie ${ }^{59}$. Por otro lado, la extensión de la linfadenectomía requerida para pacientes con cáncer de esófago sigue siendo motivo de análisis, pero se ha confirmado que la linfadenectomía por técnica mínimamente invasiva logra resultados semejantes y comparables a los obtenidos por técnica abierta, con un promedio de $17,17 \pm 9,62$ linfonodos (rango de 2-46) en comparación a la técnica abierta en distintas series con un promedio de 16-18 linfonodos (rango de 1-44). La serie de Smithers ha validado la equivalencia en ambas vías de abordaje en cuanto a la resección linfonoda ${ }^{29-31}$. En el trabajo de Luketich et al. ${ }^{53}$, la mediana de linfonodos removidos fue de 21 , la cual es comparable con las series abiertas; similarmente la tasa de resección R0 con márgenes negativos es comparable con las series de esofaguectomía por vía abierta $^{3,5,52,53,65}$

De esta manera, los resultados obtenidos en nuestra serie de pacientes sometidos a esofaguectomía, mediante técnicas mínimamente invasivas, se suma a otras publicaciones que muestran una menor tasa de morbilidad y mortalidad comparadas al abordaje abierto, sin representar un menoscabo en la calidad de la resección tumoral y la disección linfonodal. Esto ha resultado en sobrevida similares a la técnica quirúrgica abierta para las distintas etapas clínicas, siendo posible su aplicación en forma segura, incluso en estadios avanzados, siendo por lo tanto, oncológicamente equivalente a resecciones por vía abierta, con un balance de costo efectividad y excelente calidad de vida.

Por último, cabe mencionar que para la maximización de los beneficios aportados por las técnicas de cirugía mínimamente invasiva en la patología neoplásica del esófago, resulta necesaria una adecuada selección de los pacientes, encontrando su más aceptada indicación en cánceres en estadios más tempranos, aún cuando en los últimos años se han extendido sus indicaciones hacia etapas más avanzadas. Sin embargo, en opinión casi unánime hay estudios pendientes en cuanto a resultados alejados con nuevas modalidades de terapia oncológica combinada para tener resultados definitivos ${ }^{66-70}$.

\section{Referencias}

1. Pultrum BB, Bosch DJ, Nijsten MWN, Rodger SMGG, Groen $\mathrm{H}$, et al. Extended esophagectomy in elderly patients with esophageal caner: minor effect of age alone in determine the postoperative course and survival. Ann Surg Oncol. 2010;17:1572-80.

2. Stein HS, Siewert JR. Improved prognosis of resected esophageal cancer. World J Surg. 2004;28:520-5.
3. Fok M, Law S, Stipa F, Cheng S, Wong J. A comparison of transhiatal and transthoracic resection for gastroesophageal carcinoma. Endoscopy 1993;25:660-3.

4. Horstmann O, Verreet P, Becker H, Homann C, Röher HD. Transhiatal oesophagectomy compared with transthoracic resection and systematic lymphadenectomy for the treatment of oesophageal cancer. Eur J Surg. 1995;161:557-67.

5. Hulscher JB, Tijssen JG, Obertop H, van Lanschot JJ. Transthoracic versus transhiatal resection for carcinoma of the esophagus: a meta-analysis. Ann Thorac Surg. 2001;72:306-13.

6. Rindani R, Martin CJ, Cox MR. Transhiatal vs IvorLewis esophagectomy: is there a difference? Aust N Z J Surg. 1999;69:187-94.

7. Macha M, Whyte R. The current role of transhiatal esophagectomy. Chest Surg Clin North Am. 2000;10:499-517.

8. Braghetto I, Csendes A, Cardemil G, Burdiles P, Korn $\mathrm{O}$, Valladares $\mathrm{H}$. Open transthoracic or transhiatal esophagectomy versus minimally invasive esophagectomy in terms of morbidity, mortality and survival. Surg Endosc. 2006;20:1681-6.

9. Ruol A, Castoro C, Portale G, Cavellin F, Sileni VO, $\mathrm{Cagol} \mathrm{M}$, et al. Trends in management ando progresses for esophageal cancer surgery: 20 year of experience and single institution. Arch Surg. 2009;144:247-55.

10. Dubecz A, Molene D, Peters J. Modern surgery for esophageal cancer. Gastroent Clin. N.A. 2008;37:96587.

11. Plukker J, van Westreenen H. Staging in oesophageal cancer. Best Practice \& Research Clinical Gastroenterology 2006;20:877-91.

12. $3^{\circ}$ Simposium de Gastroenterología oncológica: Consenso Cáncer de esófago, Viña del Mar, 6-8 de abril de 2011, www.slago.com

13. Kato H, Miyazaki T, Nakajima M, Takita J, Kimura H, Faried A, et al. The incremental effect of positron emission tomography on diagnostic accuracy in the initial staging of esophageal carcinoma. Cancer 2005;103:14856.

14. Ursechel Sd, Vasan H. A metanalysis controlled trials tha compare neoadjuvant chemoradiation and surgery to surgery alone for resectable esophageal cancer. Am J Surg. 2003;85:533-43.

15. Kaklamans JG, Walker GP, Ferri K, Francischi B, Livingstone AS. Neoadjuvant treatment for reectable cancer of esophagus and gastric esophageal cancer junction: a metaanalysis of randomized clinical trials. Ann Surg Oncol. 2003;10:754-61.

16. Kim TJ, Kim HY, Lee KW, Kim MS. Multimodality assessment of esophageal cancer: preoperative staging and monitoring of response to therapy. Radiographics 2009;29:403-21.

17. Namasivayam V, Wang KK, Prasad GA. Endoscopic Mucosal Resection in the Management of Esophageal 
Neoplasia: Current Status and Future Directions. Clin Gastroenterol Hepatol. 2010;8:743-54.

18. Lekakos L, Karidis NP, Dimitroulis D, Tsigris C, Kouraklis G, Nikiteas N. Barrett's esophagus with highgrade dysplasia: Focus on current treatment options World J Gastroenterol. 2011;17:4174-83.

19. Gemmil EH, Mc Culloch P. Systematic review of minimally invasive resection for gastroesophageal cancer: review. Brit J Surg. 2007;94:1461-7.

20. Bollschweiler E, Baldus SE, Schröder W, Prenzel K, Gutschow C, Schneider PM, et al. High rate of lymphnode metastasis in submucosal esophageal squamouscell carcinomas and adenocarcinomas. Endoscopy 2006;38:149-56.

21. Ancona E, Rampado S, Cassaro M, Battaglia G, Ruol A, Castoro C. Prediction of lymph node status in superficial esophageal carcinoma. Ann Surg Oncol. 2008; 15:3278-88.

22. http://www.ncen.org/index.asp (Guidelines for esophageal and esophagigastric junction cancer) Version 2. 2012.

23. Braghetto I, Csendes A, Cornejo A, Amat J, Cardemil G, Burdiles P, et al. Survival of patients with esophageal cancer subjected to total thoracic esophagectomy. Rev Med Chile 2000;128:64-74.

24. Hagen JA, DeMeester SR, Peters JH, Chandrasoma P, DeMeester TR. Curative resection for esophageal adenocarcinoma: analysis of 100 en bloc esophagectomies. Ann Surg. 2001;234:520-31.

25. Hulscher JB, van Sandick JW, de Boer AG, Wijnhoven BP, Tijssen JG, Fockens P, et al. Extended transthoracic resection compared with limited transhiatal resection for adenocarcinoma of the esophagus. N Engl J Med. 2002;347:1662-9.

26. Johanson J, DeMeester TR, Hagen JA, DeMeester SR, Peters J, Oberg S, et al. On block vs transhiatal esophagectomy for stage T3N1 adenocarcinoma of the distal esophagus. Arch Surg, 2004;139:627-31.

27. Altorky NK. On block esophagectomy-The 3 fields dissection. Surg Clin N.A. 2005;85:611-9.

28. Altorky NK, Zhou KX, Stiles B, Port L, Paul S, Lee R, et al. Total number of resected lymph nodes. Ann Surg. 2008;248:221-6.

29. Rizzetto C, DeMeester SR, Hagen JA, Peyre CG, Lipham JC, DeMeester TR. En bloc esophagectomy reduces local recurrence and improves survival compared with transhiatal resection after neoadjuvant therapy for esophageal adenocarcinoma. J Thorac Cardiovasc Surg. 2008;135:1228-36.

30. Herskovic A, Martz K, al-Sarraf M, Leichman L, Brindle J, Vaitkevicius V, et al. Combined chemotherapy and radiotherapy compared with radiotherapy alone in patients with cancer of the esophagus. N Engl J Med. 1992;326:1593-8.

31. Orringer MB, Marshall B, Chang AC, Lee J, Pickens A, Lau CL. Two thousand transhiatal esophagecto- mies: changing trends, lessons learned. Ann Surg. 2007;246:363-72.

32. Chou SH, Li HP, Lee JY, Huang MF, Lee CH, Lee KW. Radical resection or chemoradiotherapy for cervical esophageal cancer? World J Surg. 2010;34:1832-9.

33. Tong DK, Law S, Kwong DL, Wei WI, Ng RW, Wong $\mathrm{KH}$. Current management of cervical esophageal cancer. World J Surg. 2011;35:600-7.

34. Sgourakis G, Gockel I, Radtke A, Musholt TJ, Timm S, Rink A, et al. Minimally invasive versus open esophagectomy: meta-analysis of outcomes. Dig Dis Sci. 2010;55:3031-40.

35. Braghetto I, Csendes A, Cardemil G, Burdiles P, Korn O, Valladares $H$. Open transthoracic or transhiatal esophagectomy versus minimally invasive esophagectomy in terms of morbidity, mortality and survival. Surg Endosc. 2006;20:1681-6.

36. Hulscher JB, Tijssen JG, Obertop H, Van Lanschot JJ. Transthoracic Versus Transhiatal Resection for Carcinoma of the Esophagus: A Meta-Analysis. Ann Thorac Surg. 2001;72:306-13.

37. Homs MY, Steyerberg EW, Eijkenboom WM, Tilanus HW, Stalpers LJ, Bartelsman JF. Single-dose brachytherapy versus metal stent placement for the palliation of dysphagia from oesophageal cancer: multicentre randomised trial. Lancet 2004;364:1497-504.

38. Fujita H, Sueyoshi S, Tanaka T, Fujii T, Toh U, Mine $\mathrm{T}$, et al. Optimal lymphadenectomy for squamous cell carcinoma in the thoracic esophagus: comparing the short-and long-term outcome among the four types of lymphadenectomy. World J Surg. 2003;27:571-9.

39. Tachibana M, Kinugasa S, Yoshimura H, Dhar DK, Nagasue N. Extended esophagectomy with 3-field lym$\mathrm{pH}$ node dissection for esophageal cancer. Arch Surg. 2003;138:1383-9.

40. Metzger R, Bollschweiler E, Vallböhmer D, Maish M, DeMeester TR, Hölscher AH. High volume centers for esophagectomy: what is the number needed to achieve low postoperative mortality? Dis Esophagus 2004; 17:310-4.

41. Urschel JD. Does the interponat affect outcome after esophagectomy for cancer? Dis Esophagus 2001;14:124-30.

42. Zhu W, Appelman HD, Greenson JK, Ramsburgh SR, Orringer MB, Chang AC, et al. A histologically defined subset of high-grade dysplasia in Barrett mucosa is predictive of associated carcinoma. Am J Clin Pathol. 2009;132:94-100.

43. Wang K, Wongkeesong M, Buttar NS. American Gastroenterological Association technical review on the role of the gastroenterologist in the management of esophageal carcinoma. Gastroenterology 2005;128:1471-505.

44. Bozio G, Baulieux J, Mabrut JY. The role of surgery in the management of Barrett's esophagus (from dysplasia to cancer). J. Visc Surg. 2011;148:19-26.

45. Hamma GB, Arya S, Markar SR. Technical factors that 
affect anastomotic integrity following esophagectomy: systematic review and meta-analysis. Ann Surg Oncol. 2013;20:4274-81.

46. Braghetto I, Cardemil G, Lanzarini E, Musleh M, Mandiola C. Impact of minimally invasive surgery in the treatment of esophageal cancer. Arq Bras Cir Dig. 2014;27:237-42.

47. Jafari MD, Halabi WJ, Smith BR, Nguyen VQ, Phelan MJ, Stamos MJ, et al. A decade analysis of trends and outcome of partial or total esophagectomy in the United States. Ann Surg. 2013;258:450-8.

48. Wouters MW, Goolker GA, Van Sandick JW. Tollenar RA. The volume-outcome relation in the surgical treatment of esophageal cancer: a systematic review and meta-analysis. Cancer 2012;118:1754-63.

49. Luketich JD, Schauer PR, Christie NA, Weigel TL, Raja S, Fernando HC, et al. Minimally invasive esophagectomy. Ann Thorac Surg. 2000;70:906-11.

50. Mu J, Yuan Z, Zhang B, Li N, Lyu F, Mao Y, et al. Comparative study of minimally invasive versus open esophagectomy for esophageal cancer in a single cancer Chin Med J. 2014;127:747-52.

51. Birkmeyer JD, Siewers AE, Finlayson EV, Stukel TA, Lucas FL, Batista I, et al. Hospital volume and surgical mortality in the United States. N Engl J Med. 2002;346:1128-37.

52. Parameswaran R, Titcomb DR, Blencowe NS, Berrisford RG, Wajed SA, Streets C, et al. Assessment and Comparison of Recovery after Open and Minimally Invasive Esophagectomy for Cancer: An Exploratory Study in Two Centers. Ann Surg Oncol. 2013:20:19707.

53. Luketich JD, Pennathur A, Awais O, Levy RM, Keeley $\mathrm{S}$, Shende M, et al. Outcomes After Minimally Invasive Esophagectomy Review of Over 1000 Patients Ann Surg. 2012;256:95-103.

54. Butler N, Collins S, Memon B, Memon MA. Minimally invasive oesophagectomy: current status and future direction. Surg Endosc. 2011;25:2071-83.

55. Verhage RJ, Hazebroek EJ, Boone J, Van Hillegersberg R. Minimally invasive surgery compared to open procedures in esophagectomy for cancer: a systematic review of the literature. Minerva Chir. 2009;64:135-46.

56. Warner S, Chang YH, Paripati H, Ross H, Ashman $\mathrm{J}$, Harold $\mathrm{K}$, et al. Outcomes of minimally invasive esophagectomy in esophageal cancer after neoadjuvant chemoradiotherapy. Ann Thorac Surg. 2014;97:439-45.

57. Watanabe M, Baba Y, Nagai Y, Baba H. Minimally invasive esophagectomy for esophageal cancer: an updated review. Surg Today 2013;43:237-44.

58. Smithers BM, Gotley DC, Martin I, Thomas JM. Comparison of the outcomes between open and minimally invasive esophagectomy. Ann Surg. 2007;245:232-40.
59. Thomson IG, Smithers BM, Gotley DC, Martin I, Thomas JM, O'Rourke P, et al. Thoracoscopic-assisted esophagectomy for esophageal cancer: analysis of patterns and prognostic factors for recurrence. Ann Surg. 2010;252:281-91.

60. Rajan PS, Vaithiswaran V, Rajapandian S, Senthilnathan P, Praveenraj P, Palanivelu C. Minimally invasive oesophagectomy for carcinoma oesophagus-Approaches and options in a high volume tertiary centre. J Indian Med Assoc. 2010;108:642-4.

61. Henriques A, Fuhro F, Godinho C, Campos AL, Waisberg J. Cervical esophagogastric anastomosis with invagination after esophagectomy. Acta Cir Bras. 2012;27:343-9.

62. Ben-David K, Sarosi GA, Cendan JC, Howard D, Rossidis G, Hochwald SN. Decreasing morbidity and mortality in 100 consecutive minimally invasive esophagectomies. Surg Endosc. 2012;26:162-7.

63. Biere SS, van Berge Henegouwen MI, Maas KW, Bonavina L, Rosman C, García JR, et al. Minimally invasive versus open oesophagectomy for patients with oesophageal cancer: a multicentre, open-label, randomised controlled trial. Lancet 2012;379:1887-92.

64. Noble F, Kelly JJ, Baile IS, Byrne JP, Underwood TJ. A prospective comparison of totally minimally invasive versus open Ivor Lewis esophagectomy. Diseases of the Esophagus 2013;26:263-71.

65. Berger AC, Bloomenthal A, Weksler B, Evans N, Chojnacki KA, Yeo CJ, et al. Oncologic efficacy is not compromised, and may be improved with minimally invasive esophagectomy. J Am Coll Surg. 2011;212:5606.

66. Benzoni E, Bresadola V, Terrosu G, Uzzau A, Cedolini $\mathrm{C}$, Intini $\mathrm{S}$, et al. Minimally invasive esophagectomy: a comparative study of transhiatal laparoscopic approach versus laparoscopic right transthoracic esophagectomy. Surg Laparosc Endosc Percutan Tech. 2008;18:178-87.

67. Dantoc MM, Cox MR, Eslick GD. Does minimally invasive esophagectomy (MIE) provide for comparable oncologic outcomes to open techniques? A systematic review. J Gastrointest Surg. 2012;16:486-94.

68. Mallipeddi MK, Onaitis MW. The Contemporary Role of Minimally Invasive Esophagectomy in Esophageal Cancer. S Curr Oncol Rep. 2014;16:374-9.

69. Takeuchi H, Kawakubo H, Kitagawa Y. Current status of minimally invasive esophagectomy for patients with esophageal cancer. Gen Thorac Cardiovasc Surg. 2013:61:513-21.

70. Lee L, Sudarsham M, Li Ch, Latimer E, Fried G, Muldr DS, et al. Cost-effectiveness of minimally invasive versus open esophagetimy for esophageal cancer. An Surg Oncol. 2013:20:3732-9. 